\author{
Martin Abraham
}

\title{
Empirische Forschung und theoretischer Fortschritt in der Familiensoziologie: Koreferat zu Johannes Huininks Beitrag
}

\author{
Empirical research and theoretical progress in family sociology:
}

Supplementary paper on Johannes Huinink's presentation

\begin{abstract}
Zusammenfassung
In diesem Beitrag wird der Frage nachgegangen, welcher Art von empirischer Forschung es bedarf, um theoretischen Fortschritt zu erzielen. In Thesenform werden vier Forschungsdesigns vorgestellt, die die „klassischen“ Forschungsdesigns fruchtbar ergänzen können: 1. der verstärkte Einsatz von Längsschnittdaten, 2. die verstärkte Betrachtung kleiner Subgruppen, 3. der Einsatz experimenteller Verfahren und schließlich 4. der Einsatz von Simulationsstudien. Der vermehrte Bezug auf diese bisher weniger gebräuchlichen Designs kann dazu beitragen, Empirie und Theorie stärker aufeinander $\mathrm{zu}$ beziehen.
\end{abstract}

Schlagworte: Forschungsdesigns, Längsschnittdaten, experimentelle Verfahren, Simulationsstudien.

\begin{abstract}
In this paper, I discuss the question what kind of empirical research should be considered necessary for achieving progress in the formation of theory. In four theses, I present research designs that could complement 'classical' research designs in a fruitful manner: first, boosting the application of longitudinal data; second, strengthening the observation of small subgroups; third, applying experimental procedures; and, fourth, applying simulation studies. A stronger emphasis on these lessemployed research designs can contribute to a stronger interrelation between empirical research and theoretical reasoning.
\end{abstract}

Keywords: research designs, longitudinal data, experimental procedures, simulation studies

Betrachtet man den Stand der empirischen Familiensoziologie, wie ihn Johannes Huinink in diesem Band skizziert, so werden schnell zwei Dinge deutlich: Erstens handelt es sich um ein Forschungsfeld, das in den letzten Jahren durch eine zunehmende Interdisziplinarität gekennzeichnet ist. Gerade in der Sichtung des empirischen Forschungsbestandes spiegelt sich die Überlappung von verschiedenen Disziplinen wie der Psychologie, der Ökonomik, der Pädagogik und eben der Soziologie wider. Genauer müsste man somit von einer empirischen Familienforschung sprechen, deren wesentliche Stärke die Betrachtung einer Fragestellung aus unterschiedlichen Blickwinkeln ist. Dass hierbei sowohl auf gemeinsam ge- 
nutzte Datensätze ${ }^{1}$ als auch von den einzelnen Disziplinen spezifisch generierte Daten zurückgegriffen werden kann, ist eine große Stärke dieses Forschungsbereiches und eröffnet die Möglichkeit, Befunde mit unterschiedlichen Daten und aus unterschiedlichen Blickwinkeln zu validieren.

Zweitens zeigt sich - zumindest aus Sicht des Autors -, dass die empirische Familienforschung nicht zuletzt aufgrund dieser Datenvielfalt eine Erfolgsstory darstellt. Obwohl naturgemäß immer noch viele Forschungslücken existieren und der sozialen Wandel immer neue Fragen generiert, besitzen wir in vielen Bereichen ein fundiertes empirisches Wissen über Entwicklung und Determinanten familialer Explananda. Am Beispiel der Scheidungsforschung wird besonders deutlich, wie das Zusammenspiel von Theorie, umfangreichen empirischen (Längsschnitt-)Daten und der Entwicklung entsprechender Methoden (Diekmann/Mitter 1984; Blossfeld/Rohwer, 1995) zu einer kumulativen Forschung geführt hat, die uns gesichertes Wissen über Ursachen und Folgen der Ehescheidung bereitstellt.

So erfolgreich sich die Teildisziplin jedoch in den letzten Dekaden entwickelt hat, lässt sich jedoch eine gewisse Stagnation im Zusammenspiel zwischen Theorie und empirischer Forschung beobachten. Seit den 1980er Jahren, in denen innovative Forschungsprogramme wie die Haushaltsökonomie (klassisch: Becker 1981) oder konstruktivistische Ansätze (z.B. Berger/Kellner 1965) erheblich die empirischen Forschungsbemühungen befördert und befruchtet haben, sind viele Fragen theoretisch noch ungelöst. Eines der vielen Beispiele stellt die geschlechtsspezifische Hausarbeitsteilung dar, die sich - wenn überhaupt - nur marginal zugunsten der Frauen geändert hat, obwohl viele zentrale Rahmenbedingungen (wie die Bildung und Erwerbstätigkeit der Frau, die Fertilitätsneigung, etc.) einem erheblichen Wandel unterworfen waren (z.B. Künzler 1995; Peuckert 2005).

Vor diesem Hintergrund wird in diesem Beitrag die Frage diskutiert, mit welcher Art von empirischer Forschung theoretischer Fortschritt entwickelt werden kann. Dahinter steht die Überzeugung, dass Theorie und Empirie sich gegenseitig bedingen und befruchten müssen, um eine (Teil-)Disziplin voranzubringen. Es werden thesenartig vier Forschungsdesigns vorgestellt, die nach Auffassung des Autors diese Aufgabe leisten können und bisher zumindest teilweise vernachlässigt wurden. Betont werden muss jedoch, dass dies als Ergänzung bisheriger „klassischer“ Forschungsdesigns (wie z.B. repräsentative Querschnittsstudien) betrachtet werden muss, die keinesfalls ausgedient haben.

\section{These 1: Verstärkter Einsatz von Längsschnittdaten}

Betrachtet man die Forschung der letzten Jahre, so hat der Einsatz von Längsschnittdaten erheblich zum Erkenntnisgewinn in der Familienforschung beigetragen. Da-

1 Vergleiche hierzu insbesondere das Sozio-oekonomische Panel (SOEP, Frick 2005), den Familiensurvey (Bender et al. 1996) und aktuell das geplante Beziehungs- und Familienpanel (siehe hierzu Brüderl et al. 2003). 
hinter steht die Erkenntnis, dass gerade die Familie als zeitabhängiger Prozess betrachtet werden muss, der sowohl vom allgemeinen sozialen und wirtschaftlichen Wandel als auch lebenslaufspezifischen Mustern geprägt ist (z.B. Mayer 1997). Dieser Prozesscharakter findet sich bisher jedoch nur unzureichend in den meist statischen Erklärungsversuchen einzelner familialer Explananda wieder. Obwohl nun im Bereich des Haushalts und der Familie sowohl eine Reihe von Paneldaten als auch retrospektive Studien existieren, sollten die Bemühungen hier weiter verstärkt werden, um die Theorieentwicklung im Hinblick auf dynamisch angelegte Erklärungen zu befördern. Insbesondere aus theoretischer Perspektive bieten gerade die großen Panelstudien (wie das SOEP) häufig zu wenige detaillierte Informationen, um spezifische Hypothesen testen zu können. Als Beispiel kann auch hier wieder die geschlechtsspezifische Hausarbeitsteilung dienen, die in den bestehenden großen Längsschnittdatensätzen nur sehr rudimentär erhoben ist. Daher sind auch in Zukunft (Längsschnitt-)Datensätze mit unterschiedlichen Schwerpunkten unabdingbar, um die erhebliche Bandbreite familienspezifischer Fragestellungen abdecken zu können.

\section{These 2: Verstärkte Betrachtung kleiner Subgruppen}

Längsschnittdaten sind zwar inzwischen eine unverzichtbare Grundlage der empirischen Familienforschung, mit ihnen können jedoch nicht alle Forschungsprobleme gelöst werden. Beispielhaft sei hier auf zwei zentrale Probleme der empirischen Familienforschung verwiesen: Erstens interessiert sich der/die Familienforscher(in) häufig für Phänomene, die nur selten vorkommen, wie z.B. die Beschreibung und Erklärung der Entwicklung neuer Familienformen. Eine derartige Entwicklung beginnt mit kleinen Fallzahlen neuer Familientypen in einer Population. Einfache Zufallsstichproben führen häufig auch bei hohem Stichprobenumfang zu einer zu geringen Anzahl von Fällen in der Subgruppe und sind daher ungeeignet, derartige Prozesse in der Anfangsphase abzubilden. Zweitens besteht häufig das Problem, dass Theorien zwar konkrete Zusammenhänge vorhersagen, diese jedoch nicht getestet werden können, da die unabhängige Variable keine oder eine zu geringe Varianz aufweist. Ein paradigmatisches Beispiel sind Effekte institutioneller oder kultureller Rahmenbedingungen auf das Handeln von Akteuren. Da Institutionen oder kulturelle Variablen in einer Population meist nicht variieren, können diese Zusammenhänge mit nationalen Samples häufig nicht untersucht werden. Es gibt allerdings immer wieder Ausnahmen, wie an zwei Beispielen kurz erläutert werden soll. Migranten bringen andere kulturelle Prägungen aus dem Gastland mit und unterliegen häufig anderen rechtlich-institutionellen Regelungen, wie z.B. ein begrenztes Arbeits- oder Aufenthaltsrecht. Beruflich selbständige Personen unterliegen nicht den institutionellen Regelungen des Arbeitsrechts und können daher z.B. eine beliebige (i.R. höhere ) Anzahl von Arbeitsstunden ,nachfragen“. Interessiert man sich für den Effekt derartiger Variablen auf unterschiedliche familiale Prozesse (wie. z.B. Fertilität, Stabilität der Partnerschaft), so können diese an derartigen Subgruppen gut untersucht werden (z.B. Nauck 1993; Abraham 2003). 
Diese Überlegungen führen zu dem Schluss, dass die Familienforschung von der verstärkten Betrachtung kleiner Subgruppen insbesondere im Hinblick auf die Theorientestung und -entwicklung profitieren könnte. Am besten wäre dies durch gezieltes Oversampling der theoretisch ausgewählten Gruppe in zufallsgenerierten Datensätzen realisierbar. ${ }^{2}$ Wünschenswert wäre insbesondere in großen Paneldatensätzen (wie das SOEP oder das geplante Beziehungs- und Familienpanel) die Möglichkeit, in einem Bus-Verfahren für zwei oder mehr Wellen mit dem gleichen Instrument die gewünschte Subpopulation ergänzend zu erheben. Auf diese Weise steht einerseits eine hinreichend große Vergleichsgruppe zur Verfügung, andererseits kann auf bereits getestete Instrumente und einen existierenden Erhebungsapparat zurückgegriffen werden. Für die Betrachtung kleiner Subgruppen spielt aber auch die qualitative Forschung eine zentrale Rolle, da mit diesen Verfahren über die häufig relativ unerforschte Subpopulation entsprechendes (Vor-)Wissen generiert werden kann.

\section{These 3: Einsatz von experimentellen Verfahren}

In engem Zusammenhang mit der vorherigen These steht die Beobachtung, dass viele für die Familienforschung interessante Phänomene empirisch schlecht zugänglich sind. Einerseits können die zu beobachtenden Konstellationen so selten sein, dass selbst ein Oversampling nicht genügend Fälle erbringt (wie der berühmt-berüchtigte VollzeitHausmann oder die Frau, die das doppelte Gehalt des Mannes nach Hause bringt), andererseits kann der alltägliche Prozesscharakter innerfamilialer Interaktionen die Beobachtung erheblich erschweren. Mit Letzterem ist beispielsweise der/die Forscher(in) konfrontiert, die sich für faktische Aushandlungsprozesse in der Partnerschaft interessiert, da eine retrospektive Erhebung von Konfliktverläufen und Aushandlungsprozessen kaum zu befriedigenden Ergebnissen führen wird.

An dieser Stelle wird die These vertreten, das experimentelle und quasi-experimentelle Verfahren in solchen Fällen gewinnbringend eingesetzt werden können. In der (familien-)psychologischen Forschung stellen Experimente natürlich schon lange ein zentrales Forschungsdesign dar (vgl. z.B. Bodenmann in diesem Heft), und in den letzten Jahren hat auch die Ökonomik das Experiment erneut entdeckt (vgl. z.B Fehr/Gächter 2000; Ockenfels 1999). In der Soziologie im Allgemeinen und in der Familiensoziologie im Besonderen stellt der Einsatz experimenteller Verfahren jedoch immer noch eine Ausnahme dar. Dies mag unter anderem in dem Umstand begründet liegen, dass das Experiment schlecht mit der für die Sozialstrukturbeschreibung wichtigen quantitativen Befragung großer Fallzahlen zu harmonieren scheint. Abgesehen von der Überlegung, dass für die Testung von Theorien nicht immer große Zufallsstichproben notwendig sind, wurden hier in den letzten Jahren quasi-

2 Wie das Design für ein derartiges Oversampling aussehen kann, ist am Beispiel der Mannheimer Scheidungsstudie zu besichtigen. In dieser retrospektiv angelegten Studie von Erstehen wurden geschiedene Ehen gezielt überrepräsentiert, um genügend Fälle für dieses im Querschnitt seltene Ereignis zu erhalten (vgl. hierzu Klein/Kopp 1999). 
experimentelle Verfahren entwickelt, die auch in der Umfrageforschung einsetzbar sind. Beispielsweise wurde der sogenannte mehrfaktorielle Survey (auch bekannt unter der Bezeichnung Vignettendesign, siehe z.B. Beck/Opp 2001; Hox et al. 1991; Sniderman/Grob 1996) bisher in der Familienforschung nicht rezipiert. Das Verfahren, mit dem simultan verschiedene unabhängige Variablen variiert und deren Effekt auf eine abhängige (Einstellungs-)Variable gemessen werden kann, scheint jedoch auch in der Familien- und Partnerschaftsforschung gewinnbringend einsetzbar zu sein (Auspurg 2005). Aber auch klassische Experimente, wie sie z.B. in der Ökonomik in den letzten Jahren äußerst erfolgreich angewendet wurden, könnten in face-toface-Interviews eingesetzt werden, um z.B. Informationen über Machtverteilung oder Altruismus in der Partnerschaft zu erhalten. Als Standardeinwand gegen experimentelle Verfahren wird schließlich immer wieder das Problem der externen Validität genannt: Sagt das Verhalten im Experiment auch tatsächlich etwas über das Verhalten im „realen Leben“ aus? Gerade hier kann die qualitative Familienforschung dazu beitragen, derartige Validitätsprobleme abzuschätzen und zu untersuchen.

\section{These 4: Einsatz von Simulationsstudien}

Wie oben bereits deutlich gemacht wurde, hat die Familiensoziologie eine Fülle von gut bestätigten Befunden über Zusammenhänge unterschiedlicher Determinanten zu bieten. Allerdings bereitet die Analyse komplexer sozialer Systeme, in denen sich unterschiedliche Variablen wechselseitig bedingen, immer noch erhebliche Probleme. Die klassische Analyse betrachtet ja meist eine abhängige Variable - z.B. die Fertilitätsneigung oder die Scheidungshäufigkeit - und versucht Erklärungen für die Unterschiede zwischen einzelnen Bevölkerungsgruppen zu finden. Dieses Vorgehen ist absolut notwendig, um Theorien entwickeln und testen zu können. Um aber langfristige Prozesse für komplexe soziale Systeme ansatzweise vorhersagen zu können - wie dies in der Politikberatung eigentlich gefordert wird - muss die Komplexität der Analyse erhöht werden. Steigt die Bildung der Frau im Zeitverlauf, hat dies nicht nur „,singuläre“ Effekte auf die Fertilität, das Heiratsalter oder die Scheidungshäufigkeit, sondern diese Faktoren werden sich jeweils auch gegenseitig beeinflussen (z.B. senkt eine wahrgenommene Scheidungswahrscheinlichkeit u.U. die Fertilitätsneigung) und haben zudem Rückkopplungseffekte auf die Ausgangsvariable (hier die Neigung zum Bildungserwerb).

Diese Komplexitätserhöhung ist mit empirischen Studien häufig nur schwer möglich (vgl. jedoch exemplarisch Beck/Hartmann 1999). Um der Forderung nach der „Prognose" der Entwicklung komplexer Systeme nachkommen zu können, werden in jüngster Zeit verstärkt (wieder) Simulationsstudien in den Sozialwissenschaften eingesetzt (Troitzsch 2000). Diese bieten den Vorteil, die Entwicklung immer noch abstrakter, aber komplexer sozialer Systeme bei simultaner Veränderung mehrerer Parameter beobachten zu können. Während nun in anderen Disziplinen - wie der Ökonomik, den Politikwissenschaften oder der Demografie - derartige Simulationsstudien bereits angewendet werden, wurde dies in der Familienforschung bisher nach einigen frühen Arbeiten (z.B. Osmond 1979) in letzter Zeit kaum rezipiert (vgl. als Ausnahmen Crosbie- 
Burnett/Elsen 1992; Chattoe 2002; Diaz et al. 2006). Dabei erscheint die Ausgangslage gerade hier besonders günstig, da die gut bestätigten Einzelergebnisse vieler empirischer Studien als Grundlage für die Konstruktion von Modellen dienen können. Wichtig erscheint jedoch die ständige Rückkopplung an die empirische Forschung, um die Aussagekraft der Simulationsmodelle einschätzen zu können. Dies vorausgesetzt, können gerade die beobachteten Unterschiede zwischen Empirie und Modell dazu beitragen, Theorien erfolgreich zu entwickeln oder zu modifizieren.

Die hier vorgeschlagenen vier Möglichkeiten, empirische Studien durch entsprechende Forschungsdesigns (noch) stärker an die Theorieentwicklung und -überprüfung zu koppeln, bilden keine abschließende Aufzählung und können sicherlich durch weitere innovative Vorschläge ergänzt werden. In jedem Fall sollten sie aber als Plädoyer dafür betrachtet werden, Empirie und Theorie stärker aufeinander zu beziehen und die Entwicklung der Familientheorie auf empirischer Basis stärker voranzutreiben. Denn letztlich erlauben es uns nur gute Theorien, die Komplexität der realen Welt handhabbar zu machen.

\section{Literatur}

Abraham, M. (2003). Die Stabilisierung von Partnerschaften durch bilaterale Investitionen. Das Beispiel der Unternehmensbesitzer. Zeitschrift für Soziologie, 32, 1, S. 50-69.

Auspurg, K. (2005). Partnerschaftliche Umzüge? Ein Vignettenexperiment zu beruflichen Umzugsentscheidungen in Partnerschaften. Diplomarbeit am Institut für Soziologie der Ludwigs-Maximilians-Universität München.

Beck, M./Opp, K.-D. (2001). Der faktorielle Survey und die Messung von Normen. Kölner Zeitschrift für Soziologie und Sozialpsychologie, 53, S. 283-306.

Beck, N./Hartmann, J. (1999). Die Wechselwirkung zwischen Erwerbstätigkeit der Ehefrau und Ehestabilität unter der Berücksichtigung des sozialen Wandels. Kölner Zeitschrift für Soziologie und Sozialpsychologie, 51, S. 655-680.

Becker, G. S. (1981). A treatise on the family. Cambridge, Mass./London: Harvard UP.

Bender, D./Bien, W./Alt, C. (1996). Anlage des Familiensurvey, Datenbasis und methodische Aspekte. In: Bien, W. (Hrsg.). Familie an der Schwelle zum neuen Jahrtausend - Wandel und Entwicklung familialer Lebensformen. Opladen: Les-ke+Budrich, S. 271-291.

Berger, P. L./Kellner, H. (1965). Die Ehe und die Konstruktion der Wirklichkeit. Eine Abhandlung zur Mikrosoziologie des Wissens. Soziale Welt, 16, S. 220-235.

Blossfeld, H.-P./Rohwer, G. (1995). Techniques of event history modeling: New approaches to causal analysis. Hillsdale, N.J.: Lawrence Erlbaum.

Bodenmann, G. (2006). Positionsbestimmungen in der Paar- und Familiensoziologie. Zeitschrift für Familienforschung. 2/2006, S. 148-174.

Brüderl, J./Esser, H./Huinink, J./Nauck, B./Walper, S. (2003). Beziehungs- und Familienentwicklung: Antrag auf Einrichtung eines DFG-Schwerpunkts SPP 1161. Chemnitz, Mannheim, München, Rostock, Bremen.

Chattoe, E. (2002). Computer simulation of family practices. In: Carling, A./Duncan, S./Edwards, R. (Hrsg.). Analysing families: Morality and rationality in policy and practice. London: Routledge, p. 268-282.

Crosbie-Burnett, M./Elsen, M. (1992). Simulated divorced and remarried families: An experiential teaching technique. Family Relations, 41, p. 54-58. 
Diaz, B. A./Fent, T./Prskawetz, A./Bernardi, L. (2006). Social influence on the transition to parenthood: An agent-based model. Vortrag auf der Tagung „Soziale Netzwerke“ der Sektion „Modellbildung und Simulation“ der Deutschen Gesellschaft für Soziologie, 31. März und 1. April 2006.

Diekmann, A./Mitter, P. (1984). Methoden zur Analyse von Zeitverläufen. Anwendung stochastischer Prozesse bei der Untersuchung von Ereignisdaten. Stuttgart: Teubner.

Fehr, E./Gächter, S. (2000). Fairness and retaliation: The economics of reciporcity. Journal of Economic Perspectives, 14, p. 159-181.

Frick, J. R. (2005). A general introduction to the German Socio-Economic Panel Study (SOEP). Design, contents and data structure [waves A-U, 1984-2004]. Arbeitspapier. Berlin: DIW.

Hox, J./Kreft, I./Hermkens, P. (1991). The analysis of factorial surveys. Sociological Methods and Research, 19, p. 493-510.

Klein, T./Kopp, J. (1999). Die Mannheimer Scheidungsstudie. In: Klein, T./Kopp, J. (Hrsg.). Scheidungsursachen aus soziologischer Sicht (S. 11-22). Würzburg: Ergon.

Künzler, J. (1995). Familiale Arbeitsteilung in der Bundesrepublik Deutschland 1988. In: Gerhardt, U. (Hrsg.). Familie der Zukunft: Lebensbedingungen und Lebensformen. Opladen: Leske + Budrich, S. 149-169.

Mayer, K. U. (1997). Lebensverläufe und sozialer Wandel. In: Mayer, K. U. (Hrsg.). Lebensverläufe und sozialer Wandel. (Sonderheft Nr. 31 der Kölner Zeitschrift für Soziologie und Sozialpsychologie). Opladen: Westdeutscher Verlag, S. 7-21.

Nauck, B. (1993). Bildung, Migration und generatives Verhalten bei türkischen Frauen. In: Diekmann, A./Weik, S. (Hrsg.). Der Familienzyklus als sozialer Prozess. Bevölkerungssoziologische Untersuchungen mit den Methoden der Ereignisanalyse (S. 309-346). Berlin: Duncker \& Humblot, S. 309-346.

Ockenfels, A. (1999). Fairness, Reziprozität und Eigennutz - Ökonomische Theorie und experimentelle Evidenz. Tübingen: Mohr Siebeck.

Osmond, M. W. (1979). The use of simulation games in teaching family sociology. The Family Coordinator, 28, p. 205-216.

Peuckert, R. (2005). Familienformen im sozialen Wandel. Wiesbaden: VS-Verlag.

Sniderman, P. M./Grob, D. B. (1996). Innovations in experimental design in attitude surveys. Annual Review of Sociology, 22, p. 377-399.

Troitzsch, K. G. (2000). Simulation in den Sozialwissenschaften. Soziologie, 2/2000, S. 33-45.

Eingereicht am: 20.05.2006

Akzeptiert am:19.06.2006

\section{Anschrift des Autors \\ Prof. Dr. Martin Abraham \\ Institut für Soziologie \\ Universität Bern \\ Lerchenweg 36 \\ CH-3012 Bern}

Email: abraham@soz.unibe.ch 
